\title{
Differences between Cervical Schwannomas of the Anterior and Posterior Nerve Roots in Relation to the Incidence of Postoperative Radicular Dysfunction
}

\author{
Yu-Ichiro Ohnishi, Koichi Iwatsuki, Toshika Ohkawa, Koshi Ninomiya, \\ Takashi Moriwaki, Toshiki Yoshimine \\ Department of Neurosurgery, Osaka University Medical School, Suita, Japan
}

\begin{abstract}
Study Design: A retrospective study.
Purpose: To assess the case files of patients who underwent surgery for cervical dumbbell schwannoma for determining the differences between schwannomas of the anterior and posterior nerve roots with respect to the incidence of postoperative radicular dysfunction.

Overview of Literature: The spinal roots giving origin to schwannoma are frequently nonfunctional, but there is a risk of postoperative neurological deficit once these roots are resected during surgery.

Methods: Fifteen patients with cervical dumbbell schwannomas were treated surgically. Ten men and 5 women, who were 35-79 years old (mean age, 61.5 years), presented with neck pain $(n=6)$, radiculopathy $(n=10)$, and myelopathy $(n=11)$.

Results: Fourteen patients underwent gross total resection and exhibited no recurrence. Follow-ups were performed for a period of 6-66 months (mean, 28 months). Preoperative symptoms resolved in 11 patients (73.3\%) but they persisted partially in 4 patients $(26.7 \%)$. Six patients had tumors of anterior nerve root origin, and 9 patients had tumors of posterior nerve root origin. Two patients who underwent total resection of anterior nerve root tumors (33.3\%) displayed minor postoperative motor weakness. One patient who underwent total resection of a posterior nerve root tumor (11.1\%) showed postoperative numbness.

Conclusions: Appropriate tumor removal improved the neurological symptoms. In this study, the incidence of radicular dysfunction was higher in patients who underwent resection of anterior nerve root tumors than in patients who underwent resection of posterior nerve root tumors.
\end{abstract}

Keywords: Cervical; Dumbbell; Schwannoma; Nerve root; Radicular dysfunction

\section{Introduction}

Spinal roots giving origin to schwannoma are frequently nonfunctional, but there is a risk of postoperative neu- rological deficit once these roots are resected during surgery. Successful surgery for cervical dumbbell schwannoma requires complete resection of tumors with maximum improvement in neurological symptoms and minimum

Received May 29, 2014; Revised Jul 28, 2014; Accepted Aug 3, 2014

Corresponding author: Koichi Iwatsuki

Department of Neurosurgery, Osaka University Medical School, 2-2 Yamadaoka, Suita, Osaka 565-0871, Japan

Tel: +81-6-6879-3652, Fax: +81-6-6879-3659, E-mail: kiwatsuki@nsurg.med.osaka-u.ac.jp 
Table 1. Clinical data of 15 patients with cervical spinal dumbbell schwannomas

\begin{tabular}{|c|c|c|c|c|c|c|c|c|c|c|c|}
\hline Case & Age/sex & Level & $\begin{array}{l}\text { Preope } \\
\text { symptom }\end{array}$ & Location & Approach & $\begin{array}{l}\text { Nerve } \\
\text { origin }\end{array}$ & $\begin{array}{l}\text { Monitoring } \\
\text { muscle }\end{array}$ & Resetion & Fusion & $\begin{array}{l}\text { Postope } \\
\text { symptom }\end{array}$ & Complication \\
\hline 1 & 58/male & $\mathrm{C} 4 / 5$ & $N, R$ & IS, For & $P$ & $\mathrm{p}$ & $D, B, T$ & $\mathrm{~T}$ & - & Resolved & - \\
\hline 2 & 69/male & C6 & $\mathrm{R}$ & IS, For & $P$ & $\mathrm{p}$ & $D, B, T$ & T & - & Resolved & - \\
\hline 3 & 58/male & $\mathrm{C} 2$ & $\mathrm{~N}, \mathrm{M}$ & IS, For & $P$ & $\mathrm{p}$ & - & $\mathrm{T}$ & - & Resolved & $\begin{array}{l}\text { Headache, } \\
\text { numbness }\end{array}$ \\
\hline 4 & 79/female & C3-5 & $M, R$ & IS, For, EA & $P$ & a & $D, B, T$ & ST & - & $\begin{array}{l}\text { Partially } \\
\text { persisted }\end{array}$ & - \\
\hline 5 & 45/male & C4 & $\mathrm{R}$ & IS, For & $P$ & $\mathrm{p}$ & $D, B, T$ & $\mathrm{~T}$ & S-plate & Resolved & - \\
\hline 6 & $35 /$ male & C4-6 & $M, R$ & IS, For, EA & $A+P$ & a & $D, B, T$ & $\mathrm{~T}$ & S-plate & $\begin{array}{l}\text { Partially } \\
\text { persisted }\end{array}$ & C5 weakness \\
\hline 7 & 72/female & $\mathrm{C} 5 / 6$ & $N, M, R$ & IS, For & P & $\mathrm{p}$ & $D, B, T$ & $\mathrm{~T}$ & LMS & Resolved & - \\
\hline 8 & 65/female & C6/7 & $M, R$ & IS, For & $A+P$ & $\mathrm{p}$ & $D, B, T, A P$ & T & $A F$ & Resolved & - \\
\hline 9 & 42/female & C6/7 & $\mathrm{M}, \mathrm{R}$ & IS, For & $P$ & a & $D, B, T, A P$ & $\mathrm{~T}$ & - & Resolved & - \\
\hline 10 & 78/male & $\mathrm{C} 7 / 8$ & $\mathrm{~N}, \mathrm{M}$ & IS, For & $P$ & a & $B, T, A P$ & $\mathrm{~T}$ & - & Resolved & - \\
\hline 11 & 64/male & C3 & $\mathrm{N}$ & IS, For, EA & $A+P$ & $\mathrm{p}$ & $D, B, T$ & $\mathrm{~T}$ & $\mathrm{AF}$ & Resolved & - \\
\hline 12 & 57/male & $\mathrm{C} 2$ & $\mathrm{~N}, \mathrm{M}$ & IS, For & $P$ & p & - & $\mathrm{T}$ & - & Resolved & - \\
\hline 13 & 70/male & C2 & $M, R$ & IS, For, EA & $\mathrm{P}$ & p & - & $\mathrm{T}$ & - & $\begin{array}{l}\text { Partially } \\
\text { persisted }\end{array}$ & - \\
\hline 14 & 75/male & C6/7 & $\mathrm{M}, \mathrm{R}$ & IS, For & $\mathrm{P}$ & a & $D, B, T, A P$ & $\mathrm{~T}$ & - & $\begin{array}{l}\text { Partially } \\
\text { persisted }\end{array}$ & Headache \\
\hline 15 & 56/female & $\mathrm{C} 6 / 7$ & M & IS, For & $\mathrm{P}$ & a & $D, B, T, A P$ & $\mathrm{~T}$ & - & Resolved & C7 weakness \\
\hline
\end{tabular}

$\mathrm{N}$, neck pain; R, radiculopathy; IS, intraspinal canal component; For, intra- or extra-foraminal canal component; $P$, posterior approach; $p$, posterior root; D, deltoid muscle; $B$, biceps muscle; T, triceps muscle; $M$, myelopathy; EA, extra-axial components; a, anterior root; ST, subtotal; S-plate, spinous process plates; $A$, anterior approach; $A P$, adductor pollicis muscle; LMS, lateral mass screw; $A F$, anterior fusion.

neurological deficits [1-5]. However, there is always a risk of postoperative neurological deficit once resection of the nerve root is performed [1,2,6-11]. It is not completely understood if the incidence of postoperative radicular dysfunction following resection is related to whether the tumor involves the anterior or posterior nerve roots. In this work, we retrospectively assessed the improvement in neurological symptoms and the incidence of neurological complications following surgery for cervical dumbbell schwannoma at our institution.

\section{Materials and Methods}

Fifteen patients underwent surgical treatment for cervical dumbbell schwannoma between March 2007 and March 2012 (Table 1). Ten men and 5 women, 35-79 years of age (mean age, 61.5 years), presented with neck pain $(n=6)$, radicular complaints $(n=10)$, and myelopathy $(n=11)$. Preoperative and postoperative assessments included magnetic resonance imaging (MRI) and computed tomography $(\mathrm{CT})$. We preoperatively assessed the vertebral arteries in all of the patients by CT angiography.

Under general anesthesia, the patients were placed in the prone position. The neck was maintained in a slightly flexed position with a Mayfield head clamp. A midline incision was extended downwards through the ligamentum nuchae. Unilateral subperiosteal exposure of the posterior spinal elements was performed in the affected side. Bilateral muscle dissection was performed if the tumor had extended up to the midline. The muscle dissection was continued laterally to expose the entire facet joint at the affected level. Hemilaminectomy and partial facetectomy were performed. The extent of lamina removal depended on the size of the intraspinal tumor component. The exposure allowed for visualization of the margin between the spinal cord and the tumor. The intraspinal tumor component was removed first. Monopolar electrical nerve root stimulation was performed. All of the roots 
from which the tumors originated were sacrificed, based on a lack of monopolar nerve stimulation response. After intradural tumor removal, the dissection was continued on the surface of the expanded root sleeve to define tumor margins. The dissection plane was bloody, given the extensive venous rete that surrounded the nerve root and VA. The tumor was followed distally along the nerve root to its lateral margin. Internal decompression was necessary for tumor margin identification. Posterior fixation was performed following medial one-half facetectomy. Tumors occupying greater than one-third of the vertebral body were removed by an anterior approach and anterior fixation was performed. Tumors occupying less than onethird of the vertebral body were removed by an anterior approach alone, and fat tissue was found to occupy the space in the vertebral body after tumor resection. External fixation was needed for 4 weeks.

Intraoperative electromyography (EMG) and electrical stimulation with compound muscle action potential (CMAP) recordings were used to identify and monitor the root tumor; a monopolar stimulation electrode was used to elicit evoked EMG responses [7]. Electrical stimulation began at $0.0 \mathrm{~mA}$ and was raised to $2 \mathrm{~mA}$ in $0.1 \mathrm{~mA}$ increments. The pulse width was $0.2 \mathrm{msec}$. EMG electrodes were placed over the deltoid, biceps, triceps, and adductor pollicis; these muscles are generally known to be innervated by the cervical roots. NIM-Response (Medtronic Inc., Tokyo, Japana) was used to stimulate the nerve root in these areas. In some cases, somatosensory evoked potentials were also recorded.

General anesthesia was achieved through intravenous administration of propofol or fentanyl, with volatile inhalation agents administered only during intubation. Muscle relaxants were used during intubation, and a minimum of 1 hour was allowed to elapse before root testing was performed. Atrophy of target muscles innervated by the cervical nerve roots involved in the schwannoma was not observed in all of the patients.

\section{Results}

Twelve patients underwent surgical resection using a posterior approach combined with hemilaminectomy and partial facetectomy (Table 1). Three of these 12 patients required posterior fixation following medial one-half facetectomy (Fig.1). Three patients were treated using a 2-stage anterior and posterior approach. Gross total resection was achieved in 14 patients. Subtotal resection was performed using a 1-stage posterior approach in the oldest patient included in our study; the surgical objective in this case was resection of intraspinal components without fusion.

The VA was preserved in all of the patients excluding 1 patient; this patient exhibited tolerance during the balloon occlusion test. The VA was sacrificed along with the embedded tumors since they adhered firmly to the VA wall. Spinal stabilization was performed using spinous process plates in 2 patients, while a lateral mass screw was used in 1 patient. When the distance from the tumor edge to the midline of spinal canal was more than $30 \mathrm{~mm}$, medial one-half facetectomy was needed, and therefore, posterior fixation was performed with spinous process plates or lateral mass screws. Anterior plate fixation was performed in 2 patients using auto-bone grafts (Fig. 2). All of the pathological examination findings were indicative of schwannoma.

We identified the tumors involving the anterior or posterior roots microsurgically and anatomically based on the anatomical position of the posterolateral sulcus and dentate ligaments. Six patients had tumors of anterior nerve root origin, and 9 patients had tumors of posterior nerve root origin. Tumor origin in the anterior root was located ventral to the dentate ligament. Sacrifice of the participating nerve was required in all of the patients. None of the patients responded to intraoperative electric monopolar nerve stimulation. Three patients developed mild deficits. One patient who underwent total resection of a C2 posterior nerve root tumor displayed postoperative numbness in the $\mathrm{C} 2$ dermatome. Two patients who underwent total resection of $\mathrm{C} 5$ and $\mathrm{C} 7$ anterior nerve root tumors displayed partial loss of strength of the deltoid and triceps muscles, respectively. None of the patients developed paralysis.

Complete tumor resection was confirmed by contrastenhanced MRI (Fig. 3). None of the patients were confined to bed following surgery, and they were followed up for a period of 6-66 months (mean, 28 months). None of the patients developed spinal instability. Successful fusion occurred in all of the cases which underwent instrumented surgery. No perioperative mortality was observed. Preoperative symptoms resolved in 11 patients (73.3\%) but they persisted partially in 4 patients (26.7\%). Four patients exhibited partial myelopathy and radiculopathy. Two patients exhibited transient headaches owing to low 

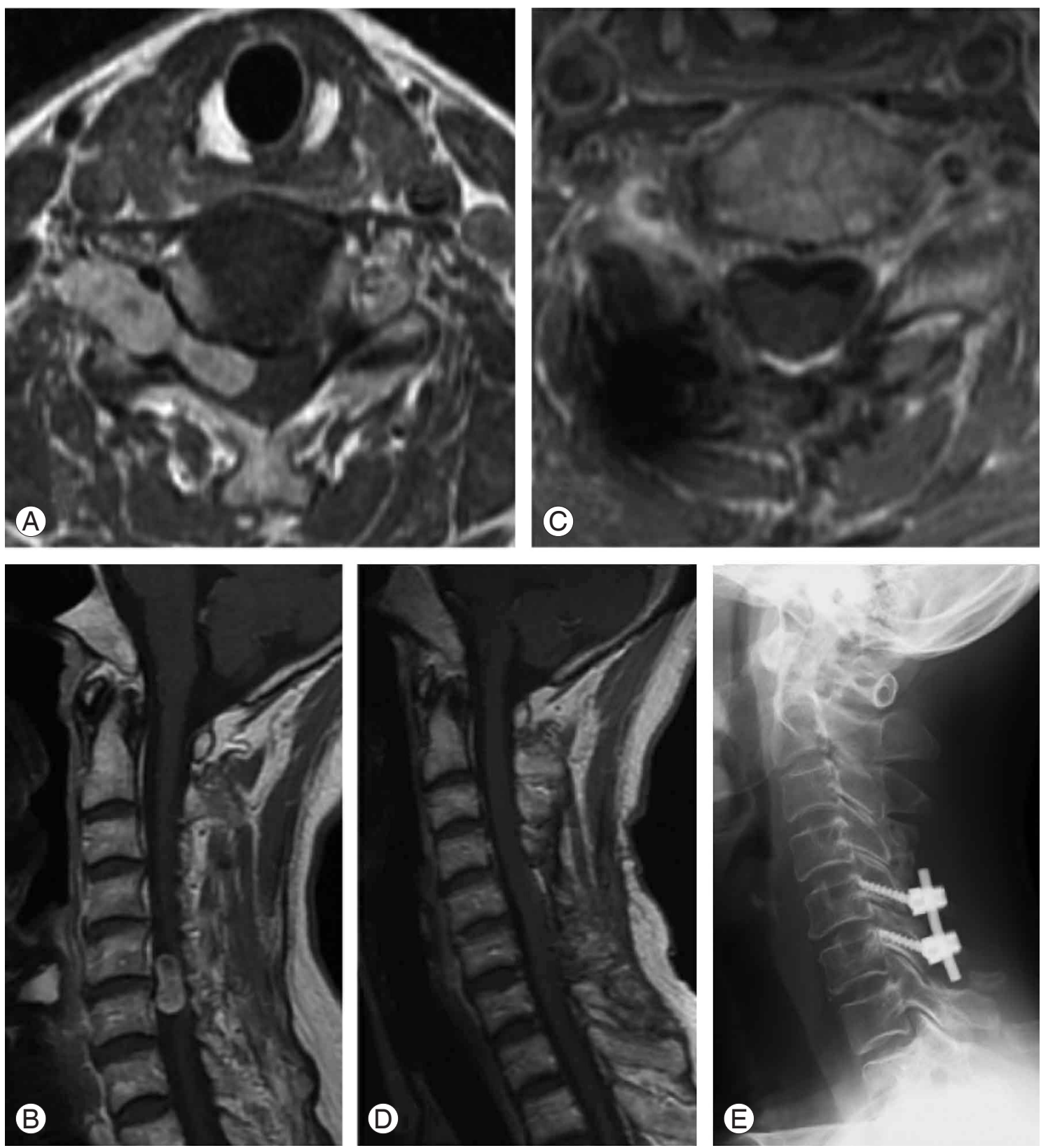

Fig. 1. Contrast-enhanced axial (A) and coronal (B) magnetic resonance (MR) images of patient no. 7 show a C5-C6 dumbbell tumor. The VA is displaced anteromedially. Postoperative contrast-enhanced axial (C) and coronal (D) MR images show complete tumor removal. Postoperative lateral roentgenogram (E) shows lateral mass screw placement. VA, vertebral arteries.

postoperative cerebrospinal fluid pressure.

\section{Discussion}

Schwannomas are benign, slow-growing tumors; therefore, excessive surgical insult and surgery-induced neurological deficits must be avoided. Most spinal schwannomas can be resected completely with or without minor postoperative deficits $[1-5,9,10]$. Several studies have reported the risk and incidence of neurological deterioration following resection of the nerve roots involved in schwannomas. Kim et al. [2] reported that 7 of 31 patients $(22.5 \%)$ developed motor or sensory deficits postoperatively. Celli et al. [1] reported that 4 of 15 (26.6\%) patients had motor weakness after resection of cervical nerve roots (C5-C8). Schultheiss and Gullotta [9] reported that 4 of 10 patients (40\%) developed motor deficits postoperatively; 2 of these patients (20\%) recovered com- 

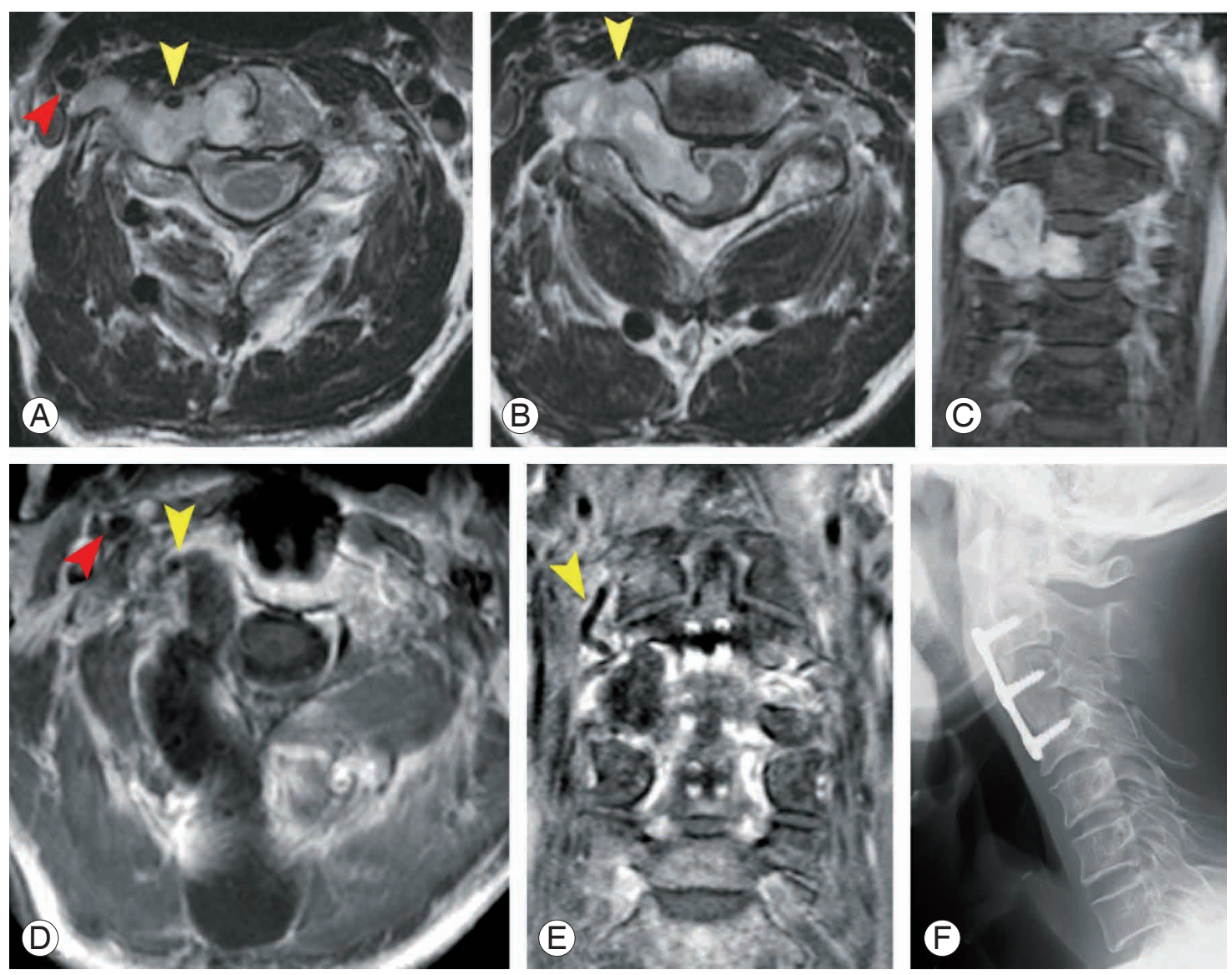

Fig. 2. Contrast-enhanced axial (A, B) and coronal (C) magnetic resonance (MR) images of patient no. 11 show a C3 dumbbell tumor. The VA is displaced anteromedially (yellow arrowhead). Contact of the tumor with the internal carotid artery can be seen (as indicated by the red arrow). Postoperative contrast-enhanced axial (D) and coronal (E) MR images show complete tumor removal. Postoperative lateral roentgenogram (F) shows anterior fixation of the iliac bone with a plate. VA, vertebral arteries.
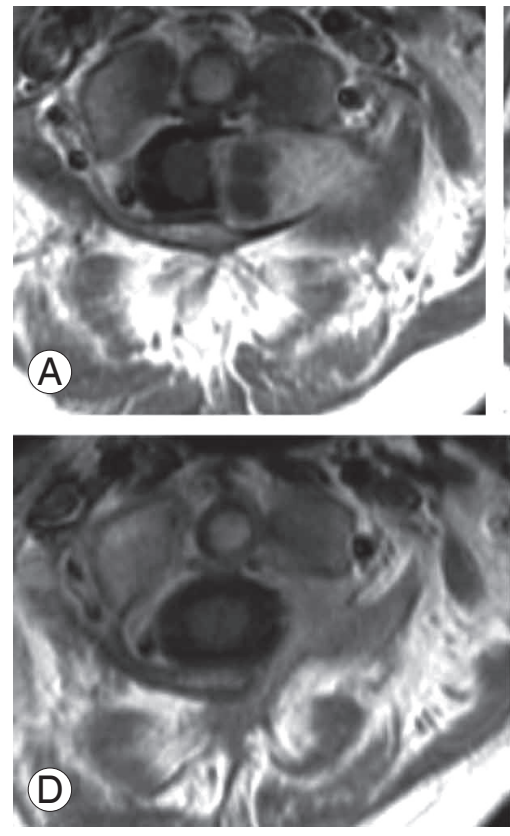
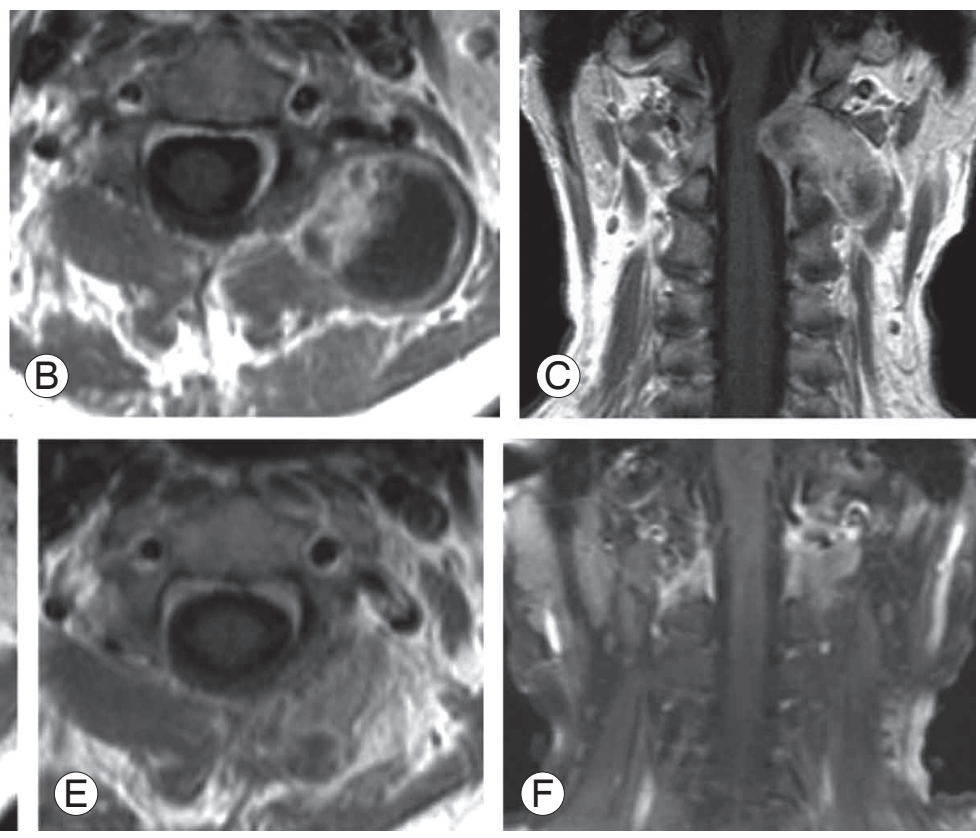

Fig. 3. Contrast-enhanced axial (A, B) and coronal (C) magnetic resonance (MR) images of patient no. 13 show a $C 2$ dumbbell tumor. Postoperative T1WI axial (D, E) and coronal (F) MR images show complete tumor removal. 
pletely and a persisting relevant motor impairment was not observed.

In this study, 3 of 15 patients developed neurological deficits. Two of 6 patients with anterior nerve root tumors $(33.3 \%)$ had minor postoperative motor weakness. Only 1 of the 9 patients with posterior nerve root tumors $(11.1 \%)$ had postoperative segmental sensory numbness. Among the patients who underwent division of the nerve root in conjunction with total resection of the schwannoma, 22\% developed neurological deficits. We classified cervical dumbbell schwannomas into intraspinal canal components, intra- or extra-foraminal canal components, and extra-axial components, indicating invasion and osteolytic expansion of the vertebral body. The development of postoperative deficits was not related to the tumor extension.

It has been reported that gradual functional denervation of the participating nerve root allows for reinnervation by adjacent nerve roots [11]. Individual dermatomes overlap to some extent [12]; Slipman et al. [13] demonstrated that resection of posterior nerve root tumors results in almost no detectable loss of skin sensibility. However, a significant number of nerve roots innervate a broad range of muscles [14]. The deltoid and triceps muscles primarily receive nerve root contributions from C5 and C7, respectively $[13,14]$. Patients with anterior nerve root tumors had a higher incidence of radicular complications compared with patients with posterior nerve root tumors. This suggests that anterior nerve roots have a more limited capacity to allow for reinnervation by adjacent nerve roots.

Triggered EMG can be recorded via electrical stimulation and evokes CMAPs in the innervated muscles [15]. Intraoperative CMAPs are obtained by submaximal stimulation and result in an all-or-none response. Chronically compressed nerve roots and nerve roots that have undergone axonotmesis require higher stimulation thresholds (>10-20 mA) [16]. Normal stimulation threshold is $0.2-5.7 \mathrm{~mA}$. In other studies, electric stimulation was raised to 5-6 $\mathrm{mA}[2,17]$. In this study, the maximum stimulation level was $2 \mathrm{~mA}$. Two patients who underwent total resection of anterior nerve root tumors showed no response to monopolar nerve stimulation. It is possible that our stimulation intensity was weak to elicit a nerve root tumor response.

Surgical approaches for cervical dumbbell tumors remain a matter of controversy. Anterolateral surgery has been proved to be useful for ventrolateral schwannoma $[3,7,8]$. Anterolateral approach offers the advantage of direct access to the lesion without retraction of the spinal cord. The disadvantage of the anterolateral approach is the possibility of injury to the VA and to the phrenic, vagus, accessory, and hypoglossal nerves. The other disadvantage is that this approach is unfamiliar to most of the spine surgeons.

Posterior approach is the standard technique for intraspinal lesions [18]. A combination of posterior approach and unilateral facetectomy has been reported for resection of cervical spine dumbbell tumors [4]. Posterior exposure provides extensive intraspinal and intraforaminal access for adequate exposure of tumor components. A posterior approach requires hemilaminectomy and facetectomy for managing laterally extended tumors. The lateral end of tumors present between muscles is deep, and it is associated with the surrounding vessels. Tumor dissection is difficult even with microsurgery. A posterior approach results in severe bleeding from the venous plexus in the foraminal portion of tumors. Hemilaminectomy and facetectomy in a posterior approach are effective in controlling bleeding from the venous plexus in the foraminal portion of tumors. When the distance from the tumor edge to the midline was more than $30 \mathrm{~mm}$, medial one-half facetectomy followed by posterior fixation was needed.

Anterior approach or combined posterior and anterior approach has been reported for cervical dumbbell schwannoma $[19,20]$. Advantages of a combined posterior and anterior approach for resection of a cervical spinal cord tumor extending into or through the foramen have been described [21,22]. Anterior fixation can be performed for treating cervical spine instability after removal of tumors that exhibit invasion and cause osteolytic expansion of the vertebral body. Tumors occupying greater than one-third of the vertebral body were removed along with anterior fixation $[7,8,23,24]$. In this study, partial facetectomy was performed with a gap in the vertebral body even if tumors occupied less than one-third of the vertebral body, and anterior fixation of the spinal column was performed.

Unilateral facetectomy results in a $20 \%-40 \%$ decrease in strength [25]. The decrease in strength occurs in $50 \%-75 \%$ facet resections in bilateral facetectomy [26]. Effects of unilateral facetectomy are considerably lesser than those of $75 \%$ bilateral facetectomy [26]. Significant 
increases in annulus stresses and segmental mobility may occur when bilateral facet resection exceeds $50 \%$ [26]. In this study, over half facetectomy and posterior fixation with a spinous process plate or a lateral mass screw were performed.

\section{Conclusions}

Our results indicated that appropriate tumor removal improved the neurological symptoms, and there was a higher incidence of radicular dysfunction in patients who underwent resection of anterior nerve root cervical dumbbell schwannomas than in patients who underwent resection of posterior nerve root cervical dumbbell schwannomas.

\section{Conflict of Interest}

No potential conflict of interest relevant to this article was reported.

\section{Acknowledgments}

We thank M. Ishihara (Osaka Rosai Hospital).

This study was supported by grants from the Funding Program for World-Leading Innovative R\&D on Science and Technology and Global COE Program from the Japanese Ministry of Education, Culture, Sports, Science, and Technology.

\section{References}

1. Celli P, Trillo G, Ferrante L. Spinal extradural schwannoma. J Neurosurg Spine 2005;2:447-56.

2. Kim P, Ebersold MJ, Onofrio BM, Quast LM. Surgery of spinal nerve schwannoma. Risk of neurological deficit after resection of involved root. J Neurosurg 1989;71:810-4.

3. Lot G, George B. Cervical neuromas with extradural components: surgical management in a series of 57 patients. Neurosurgery 1997;41:813-20.

4. McCormick PC. Surgical management of dumbbell tumors of the cervical spine. Neurosurgery 1996;38: 294-300.

5. Safavi-Abbasi S, Senoglu M, Theodore N, et al. Microsurgical management of spinal schwannomas: evaluation of 128 cases. J Neurosurg Spine 2008;9:40-7.
6. Celli P. Treatment of relevant nerve roots involved in nerve sheath tumors: removal or preservation? Neurosurgery 2002;51:684-92.

7. George B, Lot G. Neurinomas of the first two cervical nerve roots: a series of 42 cases. J Neurosurg 1995;82: 917-23.

8. George B, Lot G. Surgical treatment of dumbbell neurinomas of the cervical spine. Crit Rev Neurosurg 1999;9:156-60.

9. Schultheiss R, Gullotta G. Resection of relevant nerve roots in surgery of spinal neurinomas without persisting neurological deficit. Acta Neurochir (Wien) 1993;122:91-6.

10. Seppala MT, Haltia MJ, Sankila RJ, Jaaskelainen JE, Heiskanen O. Long-term outcome after removal of spinal schwannoma: a clinicopathological study of 187 cases. J Neurosurg 1995;83:621-6.

11. Hasegawa M, Fujisawa H, Hayashi Y, Tachibana O, Kida S, Yamashita J. Surgical pathology of spinal schwannoma: has the nerve of its origin been preserved or already degenerated during tumor growth? Clin Neuropathol 2005;24:19-25.

12. Slipman CW, Plastaras CT, Palmitier RA, Huston $\mathrm{CW}$, Sterenfeld EB. Symptom provocation of fluoroscopically guided cervical nerve root stimulation. Are dynatomal maps identical to dermatomal maps? Spine (Phila Pa 1976) 1998;23:2235-42.

13. Schirmer CM, Shils JL, Arle JE, et al. Heuristic map of myotomal innervation in humans using direct intraoperative nerve root stimulation. J Neurosurg Spine 2011;15:64-70.

14. Zhang L, Zhang CG, Dong Z, Gu YD. Spinal nerve origins of the muscular branches of the radial nerve: an electrophysiological study. Neurosurgery 2012; 70:1438-41.

15. Holland NR. Intraoperative electromyography. J Clin Neurophysiol 2002;19:444-53.

16. Holland NR, Lukaczyk TA, Riley LH 3rd, Kostuik JP. Higher electrical stimulus intensities are required to activate chronically compressed nerve roots. Implications for intraoperative electromyographic pedicle screw testing. Spine (Phila Pa 1976) 1998;23:224-7.

17. Kaneko K, Kato Y, Kojima T, Imajyo Y, Taguchi T. Intraoperative electrophysiologic studies on the functions of nerve roots involved in cervical dumbbellshaped schwannoma and their clinical utility. J Spinal Disord Tech 2006;19:571-6. 
18. Uede T, Kurokawa Y, Wanibuchi M, Ze PH, Ohtaki M, Hashi K. Surgical approach for cervical dumbbell type neurinoma: posterior approach by partial hemilaminectomy with preservation of a facet joint. No Shinkei Geka 1996;24:675-9.

19. Habal MB, McComb JG, Shillito J Jr, Eisenberg HM, Murray JE. Combined posteroanterior approach to a tumor of the cervical spinal foramen. Technical note. J Neurosurg 1972;37:113-6.

20. Iwasaki Y, Hida K, Koyanagi I, Yoshimoto T, Abe H. Anterior approach for dumbbell type cervical neurinoma. Neurol Med Chir (Tokyo) 1999;39:835-9.

21. Asazuma T, Toyama Y, Maruiwa H, Fujimura Y, Hirabayashi K. Surgical strategy for cervical dumbbell tumors based on a three-dimensional classification. Spine (Phila Pa 1976) 2004;29:E10-4.

22. Jiang L, Lv Y, Liu XG, et al. Results of surgical treat- ment of cervical dumbbell tumors: surgical approach and development of an anatomic classification system. Spine (Phila Pa 1976) 2009;34:1307-14.

23. Bruneau M, Cornelius JF, George B. Multilevel oblique corpectomies: surgical indications and technique. Neurosurgery 2007;61:106-12.

24. Pitzen T, Lane C, Goertzen D, et al. Anterior cervical plate fixation: biomechanical effectiveness as a function of posterior element injury. J Neurosurg 2003;99:84-90.

25. Cusick JF, Yoganandan N, Pintar F, Myklebust J, Hussain $\mathrm{H}$. Biomechanics of cervical spine facetectomy and fixation techniques. Spine (Phila Pa 1976) 1988; 13:808-12.

26. Voo LM, Kumaresan S, Yoganandan N, Pintar FA, Cusick JF. Finite element analysis of cervical facetectomy. Spine (Phila Pa 1976) 1997;22:964-9. 